\title{
Field efficacy and safety of fluralaner solution for administration in drinking water for the treatment of poultry red mite (Dermanyssus gallinae) infestations in commercial flocks in Europe
}

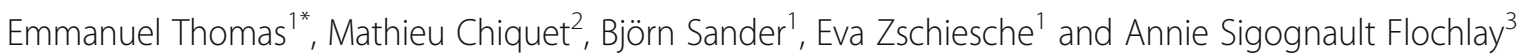

\begin{abstract}
Background: Welfare concerns, production losses caused by Dermanyssus gallinae, the poultry red mite (PRM), and widespread mite resistance to environmentally applied acaricides continue to drive an urgent need for new and effective control measures. Fluralaner is a novel systemic acaricide developed to address that need. A series of field studies was initiated to investigate the safety and efficacy of a fluralaner solution $(10 \mathrm{mg} / \mathrm{ml})$ administered in drinking water at a dose rate of $0.5 \mathrm{mg} / \mathrm{kg}$ on two occasions with a 7-day interval, for treatment of natural PRM infestations in chickens.

Methods: Blinded, negative-controlled studies were completed in Europe across eight layer, two breeder, and two replacement chicken farms. At each farm, two similar flocks were housed in similar PRM-infested units (either rooms within a building, or separate buildings) varying from 550 to 100,000 birds per unit. One unit at each farm was allocated to fluralaner treatment, administered in drinking water on Days 0 and 7. One unit remained untreated. Mite traps were placed throughout each unit on Days $-1,0$ or 1, 3, 6, 9, and 13 or 14 , then at weekly or two-weekly intervals, retrieved after $24 \mathrm{~h}$ and processed for mite counts. Efficacy at each farm was assessed by mean PRM count reductions from traps in treated units compared with those from control units. Production parameters and safety were also monitored.

Results: Efficacy was 95.3 to $99.8 \%$ on Day 3 and 97.8 to 100\% on Day 9, thereafter remaining above $90 \%$ for 56 to 238 days after treatment initiation. Post-treatment improvement in egg-laying rate was greater by 0.9 to $12.6 \%$ in the treated group at 9 of the 10 layer or breeder farms. There were no treatment-related adverse events.

Conclusion: Fluralaner administered at $0.5 \mathrm{mg} / \mathrm{kg}$ via drinking water twice, 7 days apart, was well tolerated and highly efficacious against the PRM in naturally infested chickens representing a range of production types and management systems. The results indicate that this novel treatment has potential to be the cornerstone of an integrated approach to reducing or eliminating the welfare and productivity costs of this increasingly threatening pest.
\end{abstract}

Keywords: Chicken, Clinical, Dermanyssus gallinae, Efficacy, Fluralaner, Poultry red mite

\footnotetext{
*Correspondence: emmanuel.thomas@msd.de

1 MSD Animal Health Innovation GmbH, Zur Propstei 55270, Schwabenheim,

Germany

Full list of author information is available at the end of the article
} 


\section{Background}

The poultry red mite (PRM), Dermanyssus gallinae, reduces bird welfare while causing substantial production losses (particularly in laying hens), acts as a vector of disease-causing pathogens, presents an occupational hazard for poultry-house workers and is increasingly a cause of medical concern for human populations living near poultry houses [1-10]. Recent surveys have confirmed the extremely high, increasing prevalence of PRM infestations in Europe, with an average of $83 \%$ of European layer houses infested, and up to $94 \%$ in The Netherlands, Germany and Belgium [4, 7]. Infestations with the PRM are found in all production types, from backyard or organic farms to intensive, enriched cage or barn systems [4]. The negative effects of PRM infestations on bird welfare and production are likely to be exacerbated by recent regulatory requirements which, by discontinuing use of conventional cages, may increase mite refugia while making such refugia more difficult to access with sprays and dusts. Additionally, the upcoming ban on beak trimming may also increase PRM-linked mortality [10].

As concern about the PRM grows, methods for control have become increasingly complicated. Traditional methods have relied on a range of acaricides, including carbamates, organophosphates, amidines, pyrethroids, and more recently spinosad, applied to premises and/or birds as sprays, mists and dusts [11-13]. Limitations of these approaches include: difficulties of achieving miticidal levels in all the hard-to-reach sites that harbour mites; additional stress to the birds from pesticide applications; risks of residues and pesticide exposure of workers; and the emergence of resistance to the available acaricides [11-17]. Therefore there is a long-recognized need for novel methods of reducing or even eliminating the threats that arise from PRM infestations [14].

Fluralaner, an isoxazoline compound for use as a systemic treatment for $D$. gallinae infestations, paralyses and kills mites through binding at a distinct, previously unrecognized receptor site on $\gamma$-aminobutyric acid (GABA)-gated and L-glutamate-gated chloride channels, which are widely expressed in insect and acarine central nervous and peripheral neuromuscular systems [18-20]. This mode of action is different from that of all other acaricides. A dose-ranging study in D. gallinae-infested birds demonstrated that two oral fluralaner administrations, 1 week apart, at a dose rate of approximately $0.5 \mathrm{mg} / \mathrm{kg}$ provided sustained mean mite count reductions of greater than $99 \%$ for 15 days following the first treatment, while a safety study in which birds were repeatedly treated at up to five times this dose demonstrated a wide safety margin [21, 22]. These preliminary experimental data and establishment of maximum residue levels to the satisfaction of regulatory authorities provided a basis for assessing the performance of fluralaner when administered under field conditions [23].

Studies were performed at 12 separate commercial production farms across France, Germany and Spain. The overall objective was to assess the safety and efficacy of a new fluralaner solution $(10 \mathrm{mg} / \mathrm{ml} ; 0.5 \mathrm{mg} / \mathrm{kg}$ body weight twice, 7 days apart) in the treatment of naturally acquired PRM infestations in chickens under a wide range of field conditions and management practices.

\section{Methods}

The protocol was prepared in alignment with European guidelines for testing of antiparasitic products [24]. The studies were conducted in compliance with the Good Clinical Practice Guidance Document \#85, May 9, 2001 (VICH GL9), and applicable regulatory requirements [25].

At each farm fluralaner efficacy was determined by the reductions in mean mite counts from traps placed in houses containing only fluralaner-treated birds in comparison with counts from traps placed in houses holding untreated control birds. Primary efficacy calculations were derived from two study phases: an initial phase to determine the onset of efficacy (up to 10 days duration) and a second phase in which assessments were concluded when a control group was treated for animal welfare or economic reasons.

Units for housing the birds were either separate buildings or rooms within a single building (Tables 1 and 2). Birds had access to free range on two farms. In each study all personnel, except the treatment dispenser and the farm manager who took no part in study assessments, were masked to treatment groups. At 11 of the

Table 1 Details of layer farms at the time of the first treatment

\begin{tabular}{|c|c|c|c|c|}
\hline Farm & Housing & Breed & Birds/Unit & $\begin{array}{l}\text { Age } \\
\text { (weeks) }\end{array}$ \\
\hline $01-A$ & $\begin{array}{l}\text { Barn, } 2 \text { rooms connected by a } \\
\text { not-fully hermetic ceiling }\end{array}$ & $\begin{array}{l}\text { Dekalb } \\
\text { white }\end{array}$ & 19,500 & 40 \\
\hline $02-\mathrm{A}$ & $\begin{array}{l}\text { Barn with free range, } 2 \text { rooms, } \\
\text { separated by a wall, connected } \\
\text { by a not-fully hermetic door } \\
\text { with } 2 \text { outdoor areas separated } \\
\text { by a fence }\end{array}$ & ISA Brown & 550 & $\begin{array}{l}63(\mathrm{C}), \\
54(\mathrm{~T})\end{array}$ \\
\hline 06-A & $\begin{array}{l}2 \text { rooms, separated by a } \\
\text { corridor. Enriched cages }\end{array}$ & ISA Brown & 66,000 & 40 \\
\hline 07-A & $\begin{array}{l}1 \text { building, } 2 \text { rooms, separated } \\
\text { by a corridor. Enriched cages }\end{array}$ & $\begin{array}{l}\text { Novo } \\
\text { Brown }\end{array}$ & 62,000 & 36 \\
\hline 09-A & $\begin{array}{l}2 \text { separate buildings. Enriched } \\
\text { cages }\end{array}$ & $\begin{array}{l}\text { Lohmann } \\
\text { Brown }\end{array}$ & 100,000 & $\begin{array}{l}22(T) \\
27(C)\end{array}$ \\
\hline DC1 & $\begin{array}{l}2 \text { separate buildings and free- } \\
\text { range area }\end{array}$ & $\begin{array}{l}\text { Lohmann } \\
\text { brown }\end{array}$ & $\begin{array}{l}2714(T) \\
2700(C)\end{array}$ & 46 \\
\hline DC2 & $\begin{array}{l}2 \text { separate buildings. Enriched } \\
\text { cages }\end{array}$ & $\begin{array}{l}\text { Hy-Line } \\
\text { brown }\end{array}$ & $\begin{array}{l}32,462(\mathrm{~T}) \\
18,851(\mathrm{C})\end{array}$ & 58 \\
\hline DC3 & $\begin{array}{l}2 \text { separate buildings. Enriched } \\
\text { cages }\end{array}$ & $\begin{array}{l}\text { Lohmann } \\
\text { LSL }\end{array}$ & $\begin{array}{l}54,947(T) \\
54,987(C)\end{array}$ & $\begin{array}{l}53(T) \\
54(C)\end{array}$ \\
\hline
\end{tabular}

Abbreviations: $T$ treatment group, $C$ control group 
Table 2 Details of replacement and breeder farms at the time of the first treatment

\begin{tabular}{|c|c|c|c|c|}
\hline Farm & Housing & Breed & Birds/Unit & Age (weeks) \\
\hline \multicolumn{5}{|c|}{ Replacement farms } \\
\hline $02-B$ & $\begin{array}{l}\text { Barn, } 2 \text { rooms, separated } \\
\text { by a corridor, } 2 \text { doors }\end{array}$ & Tetra Brown & $4600(\mathrm{~T}) ; 7500(\mathrm{C})$ & 13 \\
\hline $02-C$ & $\begin{array}{l}\text { Barn, } 2 \text { rooms, separated } \\
\text { by a corridor, } 2 \text { doors }\end{array}$ & $\begin{array}{l}\text { Tetra Brown, Harco Black, } \\
\text { Koenigsberger Blue and } \\
\text { Sussex }\end{array}$ & 3000 & 11 \\
\hline \multicolumn{5}{|c|}{ Breeder farms } \\
\hline 04-A & Barn, 2 separate houses & Ross 308 (cocks and hens) & 28,000 & $35(T) ; 34(C)$ \\
\hline 05-A & Barn, 2 separate houses & $\begin{array}{l}\text { RJ344 (cocks) and Ross } \\
\text { PM3 J (hens) }\end{array}$ & 8200 & 41 \\
\hline
\end{tabular}

Abbreviations: $T$ treatment group, $C$ control group

12 farms the studies were initiated during the months of the year (spring through summer) when ambient temperature and humidity favoured mite proliferation. On one farm the study began in October.

\section{Inclusion/exclusion criteria}

For a farm to be included, units for each treatment group were required to be similar, to contain the same type of hybrid bird and to be comparable regarding flock size (exceptions were made at farms 02-B and DC2), age of birds, and management practices. Chickens had to be healthy and not suffering from any concurrent disease requiring treatment and to remain at the farm for a minimum of 3 months after Day 0, except for farms producing pullets (replacements) at which observations concluded after 42 days.

On all farms each study unit was required to have a proven infestation of $D$. gallinae mobile stages determined by mite counts from traps placed between Days -14 and -7 and collected $24 \mathrm{~h}$ after placement. Depending on the the housing arrangement and bird stocking density at each farm, the number of mobile D. gallinae stages (larvae, nymphs and adults) required to establish the presence of infestation in each unit ranged from 100 to 250 mites per trap in at least $50 \%$ of traps. This criterion was the same for each unit on any one farm.

Flocks could not have been treated with products effective against $D$. gallinae in the 8 weeks before Day 0 , or in the last month with non-pharmaceutical products. Acaricide treatments with potential efficacy against $D$. gallinae were not permitted during the study unless indicated for animal welfare reasons.

\section{Study procedures}

At each farm, personnel involved in any study activities were instructed to change all handling equipment and clothing for any movement between units, and to wear single-use overall and overshoes or similar whenever entering a study unit. All working equipment was to be available in double, one set for each unit.

Feed and drinking water provision, air conditioning and stocking density followed the routine procedure of the farm. Study chickens were provided periodic veterinary care according to farm procedure, and general health observations were conducted daily in each study unit from Day -1 to Day 2, on Days 6 to 9, and then weekly until the end of the study. On study days without veterinary health observations, the farm manager documented the general impression of the flock and was to advise the dispenser immediately upon observation of any health abnormalities in study chickens.

\section{Treatment}

With the exception of farm 02-B, the unit with the highest mite infestation was allocated to the fluralaner group, the other unit to a control group that was to remain untreated for mites (unless rescue treatment was required for animal welfare or economic reasons). The targeted fluralaner dose rate was $0.5 \mathrm{mg} / \mathrm{kg}$ administered twice, with a seven-day interval between administrations (Days 0 and 7), corresponding to $5 \mathrm{ml}$ of fluralaner per $100 \mathrm{~kg}$ total body weight. Medicated water was freshly prepared and administered via a medication tank or using a dosing pump.

At each farm, within the week preceding Day 0, the inner surfaces of the drinking water distribution system of the fluralaner-treated unit were cleaned. Drinking water consumption in each of the units allocated to the fluralaner group was determined on 1 day between Days -3 and -1 (and also on Day 6 at replacement farms).

To estimate the total flock body weight to be treated, on Days -1 or $-2,24$ chickens were randomly selected from each unit to be medicated and weighed. Whenever cocks were present, the proportion of hens and cocks selected for weighing was representative of the broader population in each unit. The average chicken body weight and total flock body weight were then calculated 
based on records of the number of chickens in the flock on each day of fluralaner administration.

Immediately prior to fluralaner administration, except at farm DC2 because of risk of leakage, drinker lines were emptied, and at some farms flushed. Medicated water was prepared on each treatment day using a specific volume of the fluralaner solution $(10 \mathrm{mg} / \mathrm{ml})$ calculated according to the total estimated body weight of chickens to be treated multiplied by 0.05 (to achieve the targeted dose of $0.5 \mathrm{mg} /$ $\mathrm{kg}$ ). The product was mixed with water in a medication tank or in a stock-solution container when a dosing pump was available. Medicated water was then dispensed continuously until the medication source was empty. Visual inspection of the medicated water supply and drinking nipples verified that there were no medication obstructions or irregularities at any farm. Once empty, the medication sources were rinsed with unmedicated tap water. The pipes were then connected to the regular water distribution system to completely displace the remaining medicated water in the system. The tap-water rinse was then provided to the fluralaner-group birds.

\section{Production parameters}

The number of collected eggs was recorded daily for laying and breeding hens. Records were also available for the percentage of downgraded eggs at farms DC2 and 09-A and egg hatchability at the two breeder farms (04-A, 05-A).

All study chickens that died or were culled between Day 0 and the end of the animal phase at a farm were collected and, if possible, stored in a deep freezer. If the weekly mortality rate exceeded the usual weekly mortality rate of each unit, all cadavers or examiner-selected cadavers, were necropsied by a qualified veterinarian.

\section{Mite counts}

The mite infestation level in each unit was determined using eight to 24 traps (Fig. 1a) depending on flock and house size and production type, placed between Day -14 and -7 , depending on farm, and on Days $-1,0$ or $1,3,6,9$, and 13 or 14, then at weekly or two-weekly intervals until the end of the assessment phase. Traps were evenly distributed throughout units, with a similar distribution of traps in each corresponding unit. Traps were placed close to potential areas of mite aggregates, at fixed positions throughout the study, out of reach of the chickens, and fastened horizontally as far as possible from air ventilation systems (Fig. 1b). Traps were collected $24 \mathrm{~h}$ after placement and deep frozen for at least $48 \mathrm{~h}$ prior to shipment, or placed on dry ice for immediate shipping to a central laboratory where they were opened for counting and stage differentiation.

The mites in each trap and its plastic bag were poured into a Petri dish. Remaining mites or eggs on the cardboard of the trap or in the plastic bag were added to the mites in the dish. Mite stages were identified, differentiated and counted [26]. In traps with up to $250 \mathrm{mg}$ of $D$. gallinae (eggs and mobile stages), all mites were differentiated and counted. For traps containing more than $250 \mathrm{mg}$ of mites, a subsample of approximately $100 \mathrm{mg}$ was used for differentiation and counting.

\section{Adverse events}

An adverse event ( $\mathrm{AE}$ ) was any observation, whether or not considered product-related, that was unfavourable and unintended and occurred after the use of fluralaner. Other conditions (including mortality) commonly associated with commercial poultry husbandry (e.g. feather pecking, low level lameness, wounds, etc.) were expected and not considered to be AEs.

\section{Efficacy assessment}

The statistical units for antiparasitic efficacy evaluation were mite traps, and for production parameters the experimental unit was the poultry unit. Homogeneity of study groups was evaluated descriptively for the

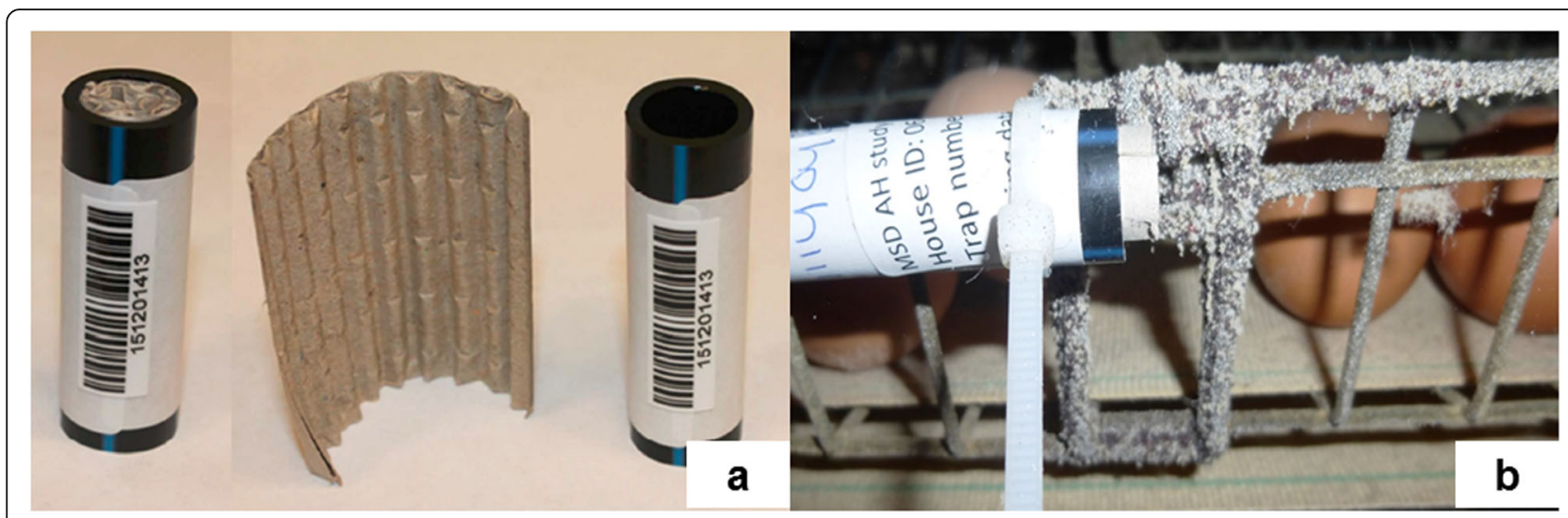

Fig. 1 Mite traps. a Black polyethylene tubes with inner and outer diameters of $12 \mathrm{~mm}$ and 16 mm, respectively, containing rolled corrugated $50 \times 60 \mathrm{~mm}$ cardboard with thickness of $1 \mathrm{~mm}$ (Avivet, the Netherlands). $\mathbf{b}$ Example of attachment of a mite trap in an area of mite aggregation 
distribution of Day -1 D. gallinae counts in each study group and production type. The statistical analysis was performed by means of the software package SAS ${ }^{\circ}$ (SAS Institute Inc., Cary, NC, USA, release 9.2).

The determination of primary efficacy was based upon the D. gallinae counts in traps collected from the units with fluralaner-treated birds compared with the control units. Percent efficacy was calculated separately for each farm for each post-treatment assessment time point using the Henderson-Tilton formula:

$$
\text { Efficacy }[\%]=\left(1-\frac{\mathrm{T}_{\text {post }}}{\mathrm{C}_{\text {post }}} \times \frac{\mathrm{C}_{\text {pre }}}{\mathrm{T}_{\text {pre }}}\right) \times 100
$$

where $\mathrm{T}_{\text {post }}$ is the mean number of mites per trap in a unit with treated chickens for each post-treatment time point; $C_{\text {post }}$ is the mean number of mites per trap in the control unit for each post-treatment time point; $T_{\text {pre }}$ is the mean number of mites per trap in a unit with treated chickens on Day $-1 ; C_{\text {pre }}$ is the mean number of mites per trap in the control unit on Day - 1 . The mean number of mites denotes the arithmetic mean of all mobile stages, i.e. larvae, nymphs (both stages together) and adults. Fluralaner was considered effective at a certain time point if efficacy was at least $90 \%$.

Chicken mortalities (including culls) in each unit during the study were compared to the pre-treatment mortalities of each group and summarized. The weekly laying rate was calculated as the number of eggs collected in relation to the number of chickens in the unit. Pre-treatment production parameters (weekly means) of each unit were compared to the post-treatment parameters, and the percent changes in treated chickens were compared to those in control chickens.

\section{Results}

There were eight layer farms, two breeding farms and two replacement farms enrolled into the studies. The breeds and ages of birds in each group (treated or control) were the same or generally similar within each farm, with the greatest difference in age of birds at a layer farm (02-A) (Tables 1 and 2).

\section{Mite count reduction}

On all sites, by Day 14 efficacy was greater than $99 \%$, remaining at a level of at least $90 \%$ through the end of the production cycle (approximately three to 8 months, farms DC2 and 06-A); until mite regrowth in the treated unit (approximately two to 6 months, farms DC3, 01-A, 02-A, 07-A, 09-A), or until the study was terminated because of declining mite counts in the control unit (4 months, DC1) (Tables 3 and 4; Fig. 2). For animal welfare reasons, a supplementary acaricide treatment (spinosad; Elector, Elanco) of the control unit was provided on Week 4 on Farm 06-A, Week 9 on Farm 01-A, and Week 20 on Farm 09-A, and (a non-chemical product) on Farm DC3 on Weeks 11, 12 and 15. Despite these acaricide treatments, mite counts from control units remained at levels that were sufficiently high to allow extended comparisons with the fluralaner-treated group beyond the dates of these treatments. These extensions were then concluded at either the end of the production cycle (DC3, 06-A) or when mite regrowth was apparent in traps from the treated unit (01-A, 09A). On this basis, mean mite count reductions in the units in which birds received fluralaner continued to exceed $90 \%$ (up to $100 \%$ ) for at least 56 days and up to 238 days (Tables 3 and 4; Fig. 3a). The post-Day 56 decline in effectiveness at Farms 01-A and 02-A was attributed to inadequate separation of the fluralaner-treated group units from the respective control units, leading to a rapid re-infestation of the treated unit (Fig. 3a, b).

At the two breeder farms, in the units in which birds were treated with fluralaner the mite reduction exceeded 99\% from Day 6 until Week 14. At Farm 04-A this reduction in mite counts was $100 \%$ on Weeks 5 through 8 , and on Farm 05-A was 100\% at Weeks 2, 4, 8, 10 and 18 (Fig. 4). Mean mite trap counts increased in the treated unit at Farm 04-A after Week 16, while high efficacy was maintained until the end of the study in Week 20 (Day 140) at farm 05-A. At the two replacement chicken (pullet) farms the fluralaner treatment resulted in the mite reduction exceeding 95\% from Day 3 until end of the study at week 6 (Day 42) (Fig. 4).

Hatched eggs of $D$. gallinae produce motile but nonfeeding larval stages which were also assessed from the collected traps. Across farms, overall reductions in larvae detected in traps had been reduced by $76.9 \%$ by Day 3 and $99.9 \%$ by Day 9 .

\section{Production assessments}

Across all study farms, the weekly mortality rates were comparable between treatment groups before and after Day 0. At one farm (DC1) there was a $0.04 \%$ increase in mortality in the fluralaner-treated group due to a colibacillosis outbreak, diagnosed at necropsy and by bacteriology.

In layers allocated to the fluralaner group, pretreatment mean weekly laying rate ranged from $-5.8 \%$ lower to $4.1 \%$ higher than in hens allocated to the control group (Table 5). Post-treatment improvement in egg laying was greater in the fluralaner-treated group at all layer farms, with the difference from the control group ranging from 0.9 to $12.6 \%$. On the two breeder farms, the difference for fluralaner compared to controls was $-2.2 \%$ and $5.7 \%$. An observation reported from layer farms was that control-group chickens would sometimes avoid laying eggs in infested nests. At farms DC2 and 09-A, the percent of downgraded eggs was also 
Table 3 Initial mite counts per unit and percentage mite efficacy on layer farms

\begin{tabular}{|c|c|c|c|c|c|c|c|c|}
\hline \multirow[t]{2}{*}{ Farm } & \multicolumn{2}{|c|}{ Mean mite counts, Day -1 } & \multicolumn{6}{|c|}{ Efficacy (\%) on days after the first fluralaner administration } \\
\hline & Treated & Control & 0 & 1 & 3 & 9 & 14 & Last day with $>90 \%$ efficacy \\
\hline $01-\mathrm{A}$ & 568 & 409 & 50.7 & nd & 96.6 & 97.8 & 99.3 & 90.7 (Day 63) \\
\hline $02-\mathrm{A}$ & 2138 & 1673 & 74.8 & nd & 98 & 99.7 & 99.8 & 97.5 (Day 56) \\
\hline 06-A & 2245 & 758 & 56.8 & nd & 99.4 & 100 & 100 & $95.4\left(\right.$ Day $238^{\mathrm{a}}$ ) \\
\hline $07-A$ & 196 & 162 & 26 & nd & 95.3 & 100 & 99.8 & 93.6 (Day 167) \\
\hline 09-A & 979 & 694 & 54.9 & nd & 99.4 & 100 & 100 & 96.5 (Day 126) \\
\hline DC1 & 2250 & 751 & - & 99.7 & 99.8 & 100 & 100 & 100 (Day 119) \\
\hline DC2 & 1610 & 367 & - & 88.7 & 96.9 & 99.6 & 99.9 & 100 (Day $89^{a}$ ) \\
\hline DC3 & 1313 & 1194 & - & nd & 96.0 & 99.9 & 99.9 & 98.7 (Day 133) \\
\hline
\end{tabular}

Abbreviation: $n d$ not determined

${ }^{a}$ End of the production cycle

recorded, with an overall post-treatment compared to pre-treatment improvement in the treated group of 3.4\% and $1.1 \%$, respectively.

At Farm 05-A, the overall hatchability rate over the complete laying period was higher in the fluralanergroup unit (78.7\%) than in the control unit (75.8\%). At the other breeder farm (04-A), the difference in hatchability rates between the treated and control groups was identical pre- and post-treatment.

Apart from a colibacillosis outbreak at farm DC1 7 weeks following fluralaner treatment initiation, there were no abnormal general health observations in treated birds. There were no treatment-related AEs.

\section{Discussion}

Parasitic stages of $D$. gallinae spend only about 1 hour obtaining a blood meal and approximately $23 \mathrm{~h}$ in the environment, so that removing traps $24 \mathrm{~h}$ after placement would allow adequate time for trap counts to be a reliable indicator of the level of challenge in a poultry house. The trap methodology has been reported previously and has been validated as a robust method to quantify mite infestations of poultry, showing that the number of mites collected in traps can be statistically correlated to the mite population in a cage [27, 28].

Once collected, the traps were deep frozen to ensure that the mites were killed, thereby avoiding the formation of mite agglomerates that would have made counting unreliable. The resulting mite counts demonstrate that a substantial mite challenge of the untreated control birds was maintained throughout all but one of the study farms, despite, in a number of cases, repeated acaricide spray treatments.

The $90 \%$ efficacy threshold selected to determine the duration of mite population control aligns with efficacy thresholds accepted by veterinary regulatory agencies for assessing ectoparasite control products [24]. On this basis, the mite population control (i.e. > 90\% reduction in mite counts) provided by the fluralaner treatment was of a rapid onset, almost complete and sustained for an extended period on each farm, regardless of production type or drinking water system. In every treated unit there was a dramatic reduction of up to $100 \%$ in mite counts.

The major factor contributing to the resurgence of mite counts in units with the chickens receiving fluralaner was the reliability, or lack thereof, of the separation of the treated and untreated groups. The presence of a non-hermetic door, fence, or ceiling was associated with a faster mite regrowth in the treated unit than when the groups were more substantially separated, as in units in two separate buildings. Because of the protocol requirement for an untreated control unit in close proximity to a treated unit, movement of study personnel and equipment, including conveyors, between the 2 units

Table 4 Initial mite counts per unit and percentage mite efficacy on replacement and breeder farms

\begin{tabular}{|c|c|c|c|c|c|c|c|}
\hline \multirow[t]{2}{*}{ Farm } & \multicolumn{2}{|c|}{ Mean mite counts, Day -1} & \multicolumn{5}{|c|}{ Efficacy (\%) on days after the first fluralaner administration } \\
\hline & Treated & Control & 0 & 3 & 9 & 14 & Last day with $>90 \%$ efficacy \\
\hline \multicolumn{8}{|c|}{ Replacements } \\
\hline 02-B & 430 & 998 & 69.2 & 95.3 & 100 & 99.7 & $96.0\left(\right.$ Day $\left.42^{a}\right)$ \\
\hline $02-C$ & 1974 & 347 & 15.1 & 96 & 99.9 & 100 & $99.6\left(\right.$ Day $\left.42^{\mathrm{a}}\right)$ \\
\hline \multicolumn{8}{|c|}{ Breeders } \\
\hline 04-A & 1148 & 521 & 71 & 98.9 & 100 & 100 & 94.5 (Day 112) \\
\hline 05-A & 9009 & 3364 & 65.2 & 99.8 & 100 & 100 & 99.9 (Day $140^{\mathrm{a}}$ ) \\
\hline
\end{tabular}

${ }^{\mathrm{a}}$ End of the production cycle or transfer to another farm 


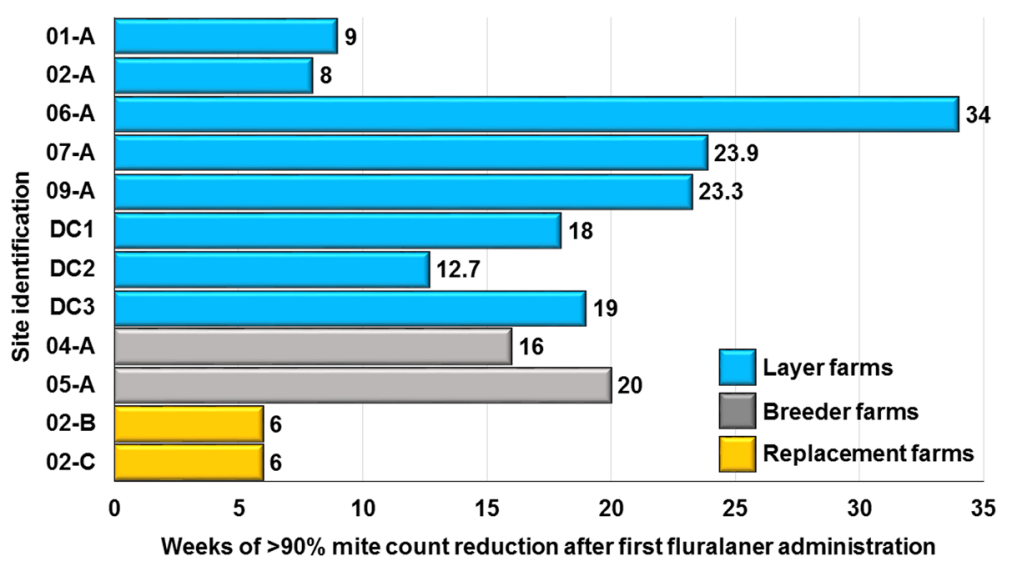

Fig. 2 Duration of mite population control in fluralaner-treated units per farm (last timepoint with mite reduction > 90\%). Sites 01-A and 02-A had inadequate separation of the treatment groups, resulting in increased risk of mite cross-contamination between units, leading to termination of study assessments. At farms 06-A, DC2, 05-A, 02-B and 02-C assessments were concluded at the end of the production cycle or transfer of chickens to another farm

(regardless of best efforts at protocol adherence and the rigour with which hygiene was practiced) would have greatly increased the risk of mite cross-contamination to the treated units. The results reported here therefore represent a worst-case scenario, as under normal commercial conditions all houses or rooms which are adjoining or in close proximity would be treated simultaneously, thereby substantially reducing or eliminating this risk of cross-contamination.

The rapid removal of adults from the population prevents additional egg production by female mites. As eggs that were present before treatment hatch within two to 3 days under favorable conditions, the emerging larvae are progressively killed after they mature to the nymphal stages and begin feeding [14]. The second fluralaner administration, 1 week after the first, kills any mites originating from eggs present at the time of the first treatment. Therefore the fluralaner treatment regimen provides the opportunity to provide a substantial and sustained reduction, or total elimination of mites from a production system. Moreover, as the PRM prevalence in laying hens is at least $80 \%$ in most of the major European egg-producing countries [7], the treatment of pullets just before they are transferred may be a valuable tool in eliminating an important source of reinfestations, and in reducing the overall prevalence of PRM in layer facilities.

The results in our studies are consistent with the linkage of PRM infestation with welfare and productivity losses, as the acaricidal efficacy of fluralaner and the resulting relief of mite-infested birds was reflected by an overall increase in laying rate in the treated groups, and a reduction in the percentage of downgraded eggs. An interesting finding
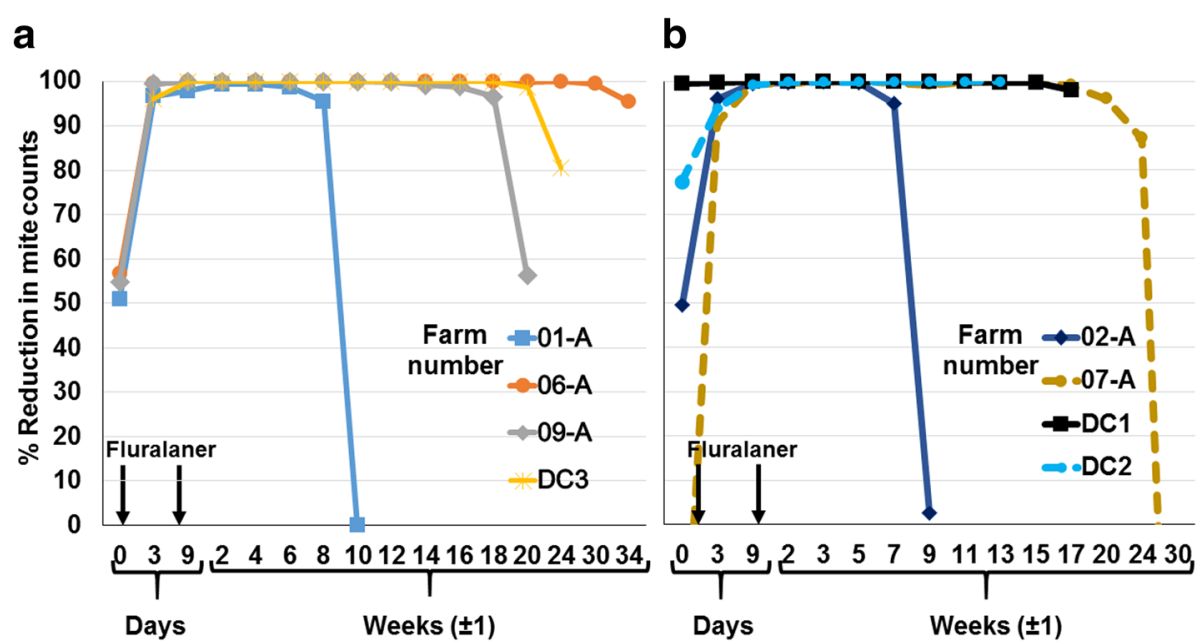

Fig. 3 Mite count reductions from fluralaner-treated units at layer farms. a Sites at which rescue-treatments were administered to control units. b Sites that did not have rescue-treatment of control birds 


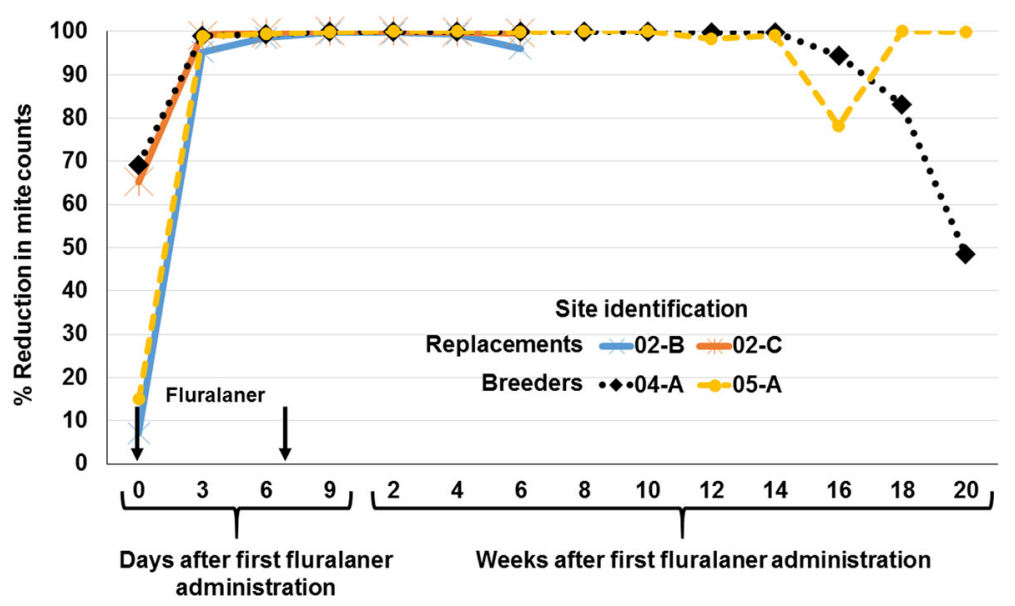

Fig. 4 Mite count reductions from fluralaner-treated units at the replacement (pullet) and breeder farms. The decline in efficacy at Week 16 on site 5-A was attributed to a decline in mite counts in the control group, rather than being caused by a resurgence in mite population in the unit housing fluralaner-treated chickens

was that in some cases, under conditions of mite challenge layers avoided their nests. Additionally, increased hatchability rates were apparent in the treated group at one of the two breeder farms. These numerical improvements in laying rates appear sufficiently promising to generate further investigation into the production benefits of fluralaner medication of PRM-infested poultry farms.

This is the first study in which the potential value of an isoxazoline has been demonstrated in food-producing animals, and the first report of successful elimination of D. gallinae from birds maintained under commercial conditions. Administration of fluralaner to the birds in this study followed the establishment of maximum residue limits for fluralaner that were adopted by the European Commission [23].

Consistent with findings from the use of fluralaner in dogs and cats, the tolerability of the fluralaner treatment

Table 5 Difference in average weekly laying rate (treated control) pre- and post-treatment on layer and breeder farms

\begin{tabular}{lllll}
\hline & Farm & Pre-treatment (\%) & Post-treatment (\%) & Difference (\%) \\
\hline Layers & 01-A & +0.43 & +1.33 & +0.90 \\
& $02-A$ & +4.05 & +16.63 & +12.58 \\
& 06-A & +0.08 & +1.15 & +1.07 \\
07-A & -0.58 & +1.45 & +2.03 \\
09-A & -1.00 & +4.70 & +5.70 \\
DC1 & -0.40 & +0.50 & +0.90 \\
DC2 & -1.30 & -0.40 & +0.90 \\
& DC3 & +3.20 & +5.10 & +1.90 \\
Breeders & 04-A & -1.08 & -3.24 & -2.16 \\
& $05-A$ & -5.83 & -0.16 & +5.67 \\
\hline
\end{tabular}

${ }^{a}$ Although the units were comparable in protocol designated characteristics, the control unit had a history of better performance relative to the treated unit in the present set of studies was excellent. There were no treatment-related AEs following the treatment of approximately 355,000 chickens across 12 farms.

\section{Conclusion}

The administration of fluralaner solution $(10 \mathrm{mg} / \mathrm{ml})$ given orally via drinking water at a dosage of $0.5 \mathrm{mg} / \mathrm{kg}$ body weight on two occasions with a seven-day interval, was well tolerated and highly effective against the poultry red mite (D. gallinae) in naturally infested chickens across a range of production types and management systems. By Day 14, mite count reductions in fluralanertreated birds was greater than $99 \%$, continuing at a reduction of least $90 \%$ for up to 8 months after treatment. The results indicate that this formulation of fluralaner has potential to be the cornerstone of an integrated approach to reducing or eliminating the welfare and productivity costs of this increasingly threatening pest.

\section{Abbreviations}

AE: adverse event; $C$ : untreated control group; $C_{\text {post }}$ : mean number of mites per trap in a control unit for each post-treatment time point; PRM: poultry red mite (Dermanyssus gallinae); T: fluralaner-treated group; $T_{\text {post: }}$ mean number of mites per trap in a unit with treated chickens; VICH: International Cooperation on Harmonization of Technical Requirements for registration of Veterinary Medicinal Products

\section{Acknowledgements}

The authors would like to thank Roland Bronneberg and Berrian Lammers (Avivet) for conducting the mite counting and for their support in the preparation of the study design, Thierry Pobel for monitoring the study farms in Spain, the field veterinarians having participated in the study, Sabrina Fuhrmann for conducting the data management and Dr. Bill Ryan (Ryan Mitchell Associates, LLC) for assistance with the manuscript.

Funding

The study was funded by MSD Animal health. 


\section{Availability of data and materials}

The data generated or analysed during these studies are included partially in this published article. They are the property of the sponsor and will not be further provided.

\section{Authors' contributions}

ET organized the studies. MC and BS monitored the study farms. EZ conducted the statistical analysis of the results. ASF was involved in the study design and in the interpretation of the results. All authors revised and approved the final manuscript.

\section{Ethics approval and consent to participate}

Informed consent was provided by the farm owner (or designate) at each farm.

\section{Consent for publication}

Not applicable.

\section{Competing interests}

ET, MC, BS and EZ are employees of MSD Animal Health. ASF is an employee of Merck Animal Health.

\section{Publisher's Note}

Springer Nature remains neutral with regard to jurisdictional claims in published maps and institutional affiliations.

\section{Author details}

'MSD Animal Health Innovation GmbH, Zur Propstei 55270, Schwabenheim, Germany. ${ }^{2}$ MSD Animal Health Innovation SAS, 7 rue Olivier de Serres, CS 67131, 49071 Angers Technopole, Beaucouzé, France. ${ }^{3}$ Merck Animal Health, 2 Giralda Farms, Madison, NJ, USA.

Received: 7 March 2017 Accepted: 18 September 2017

Published online: 09 October 2017

\section{References}

1. Cosoroaba I. Massive Dermanyssus gallinae invasion in battery-husbandry raised fowls. Rev Med Vet-Toulouse. 2001;152:89-96.

2. Van Emous R. Wage war against the red mite! Poultry Int. 2005:44:26-33.

3. George DR, Finn RD, Graham KM, Mul MF, Maurer V, Moro CV, et al. Should the poultry red mite Dermanyssus gallinae be of wider concern for veterinary and medical science? Parasit Vectors. 2015:25(8):178.

4. Sparagano OAE, George DR, Harrington DWJ, Giangaspero A. Significance and control of the poultry red mite Dermanyssus gallinae. Annu Rev Entomol. 2014;59:447-66.

5. Kowalski A, Sokol R. Influence of Dermanyssus gallinae (poultry red mite) invasion on the plasma levels of corticosterone, catecholamines and proteins in layer hens. Pol J Vet Sci. 2005;12:231-5.

6. Sommer D, Heffels-Redmann U, Köhler K, Lierz M, Kaleta EF. Role of the poultry red mite (Demanyssus gallinae) in the transmission of avian influenza A virus. Tierärztliche Praxis Grosstiere. 2016;1:47-54

7. Mul M. Fact sheet: The poultry red mite, Dermanyssus gallinae (De Geer, 1778) A small pest that packs a big punch. 2013. https://www.researchgate. net/publication/258553789_Fact_sheet_Poultry_Red_Mite_in_Europe. Accessed Sept 2017

8. Cafiero MA, Camarda A, Circella E, Santagada G, Schino G, Lomuto M. Pseudoscabies caused by Dermanyssus gallinae in Italian city dwellers: a new setting for an old dermatitis. J Eur Acad Dermatol Venereol. 2008; 22:1382-3.

9. Cafiero MA, Galante D, Camarda A, Giangaspero A, Sparagano O. Why dermanyssosis should be listed as an occupational hazard. Occup Environ Med. 2011;68:628.

10. COREMI. Memorandum of understanding: Memorandum of Understanding for the implementation of a European Concerted Research Action designated as COST Action FA1404: Improving current understanding and research for sustainable control of the poultry red mite Dermanyssus gallinae (COREMI), COST 036/14, 15 May 2014. https://e-services.cost.eu/ files/domain files/FA/Action FA1404/mou/FA1404-e.pdf . Accessed Sept 2017
11. Abbas RZ, Colwell DD, lqbal Z, Khan A. Acaricidal drug resistance in poultry red mite (Dermanyssus gallinae) and approaches to its management. Worlds Poult Sci J. 2014;70:113-24.

12. Marangi M, Cafiero MA, Capelli G, Camarda A, Sparagano OAE, Giangaspero A. Evaluation of the poultry red mite, Dermanyssus gallinae (Acari: Dermanyssidae) susceptibility to some acaricides in field populations from Italy. Exp Appl Acarol. 2008;18:11-8

13. George DR, Shiel RS, Appleby WG, Knox A, Guy JH. In vitro and in vivo acaricidal activity and residual toxicity of spinosad to the poultry red mite, Dermanyssus gallinae. Vet Parasitol. 2010;173:307-16.

14. Chauve C. The poultry red mite Dermanyssus gallinae (De Geer, 1778): current situation and future prospects for control. Vet Parasitol. 1998;79:239-45.

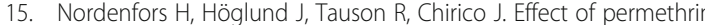
impregnated plastic strips on Dermanyssus gallinae in loose-housing systems for laying hens. Vet Parasitol. 2001;102:121-31.

16. Marangi M, Morelli V, Pati S, Camarda A, Cafiero MA, Giangaspero A. Acaricide residues in laying hens naturally infested by red mite Dermanyssus gallinae. PLoS One. 2012;7:e31795.

17. Beugnet F, Chauve C, Gauthey M, Beert L. Resistance of the red poultry mite to pyrethroids in France. Vet Rec. 1997;140:577-9.

18. Ozoe Y, Asahi M, Ozoe F, Nakahira K, Mita T. The antiparasitic isoxazoline A1443 is a potent blocker of insect ligand-gated chloride channels. Biochem Biophys Res Commun. 2010;391:744-9.

19. Gassel M, Wolf $\mathrm{C}$, Noack S, Williams H, Il T. The novel isoxazoline ectoparasiticide fluralaner: selective inhibition of arthropod $\gamma$-aminobutyric acid- and L-glutamate-gated chloride channels and insecticidal/acaricidal activity. Insect Biochem Mol Biol. 2014;45:111-24.

20. Casida JE. Golden age of RyR and GABA-R diamide and isoxazoline insecticides: common genesis, serendipity, surprises, selectivity, and safety. Chem Res Toxicol. 2015;28:560-6.

21. Heckeroth AR, Zoller H, Flochlay-Sigognault A, Huyghe B. Use of isooxazoline derivatives for the treatment or prevention of arthropod infestations in poultry. Patent WO. 2015:2015091900:A1.

22. Prohaczik A, Menge M, Huyghe B, Flochlay-Sigognault A, Le Traon G. Safety of fluralaner oral solution, a novel systemic antiparasitic treatment for chickens, in laying hens after oral administration via drinking water. Parasit Vectors. 2017:10:363.

23. European Medicines Agency. European public MRL assessment report (EPMAR) Fluralaner (poultry). Available at: http://www.ema.europa.eu/docs/ en_GB/document_library/Maximum_Residue_Limits_-_Report/2017/02/ WC500221753.pdf. Accessed Sept 2017.

24. European Medicines Agency. Directive 81/852/EEC as amended. Demonstration of efficacy of Ectoparasiticides. 1994. http://www.ema. europa.eu/docs/en_GB/document_library/Scientific_guideline/2009/10/ WC500004662.pdf. Accessed Sept 2017

25. European Medicines Agency, Committee for Medicinal Products for Veterinary Use, 2000. Guideline on Good Clinical Practices. VICH Topic GL9. http://www.ema.europa.eu/docs/en_GB/document_library/Scientific guideline/2009/10/WC500004343.pdf. Accessed Sept 2017.

26. Taylor MC. Veterinary Parasitology. 3rd ed. Oxford: Blackwell Publishing Ltd.: 2007. p. 528-9.

27. Meyer-Kühling B, Pfister K, Müller-Lindloff J, Heine J. Field efficacy of phoxim 50\% (Byemite) against the poultry red mite Dermanyssus gallinae in battery cages stocked with laying hens. Vet Parasitol. 2007;147:289-96.

28. Lammers GA, Bronneberg RGG, Vernooij JCM, Stegeman JA. Experimental validation of the AviVet trap, a tool to quantitatively monitor the dynamics of Dermanyssus gallinae populations in laying hens. Poult Sci. 2017;96(6): 1563-72. 